\title{
Identification of a long non-coding RNA signature for predicting prognosis and biomarkers in lung adenocarcinoma
}

\author{
XIAOLIN YU ${ }^{1}$ and YANXIA ZHANG ${ }^{2}$ \\ ${ }^{1}$ Department of Internal Medicine of Traditional Chinese Medicine, Beijing University of Chinese Medicine; ${ }^{2}$ Department \\ of Respiratory, Dongfang Hospital, Beijing University of Chinese Medicine, Beijing 100078, P.R. China
}

Received July 26, 2019; Accepted January 16, 2020

DOI: $10.3892 / \mathrm{ol} .2020 .11400$

\begin{abstract}
Long non-coding RNAs (lncRNAs) have a number of functions in various cellular processes and are potential prognostic factors for lung adenocarcinoma (LUAD). A gene risk model could provide novel evidence to improve the prediction of overall outcomes and provide more potential biomarkers. The present study aimed improve a previously published method of gene signature construction to make it more robust and accurate. The lncRNA expression profiles from 594 patients with LUAD were obtained from The Cancer Genome Atlas (TCGA) database and samples were divided into high- and low-risk groups based on median risk scores calculated using a prognosis-related risk score formula. Univariate Cox regression, least absolute shrinkage and selection operator algorithm and multivariate Cox regression were performed to construct a gene signature based on the differentially expressed IncRNAs in patients with LUAD. The robustness and accuracy of the present model was assessed using area under the calculated curves (AUC) and Kaplan-Meier (K-M) survival analysis of the high- and low-risk cohorts. Potential biomarkers associated with survival status were then identified using $\mathrm{K}-\mathrm{M}$ survival analysis and potential biomarker functions were predicted using enrichment analysis of co-expressed mRNAs. The gene signature constructed contained 44 lncRNAs. The AUCs for 3- and 5-year survival with the model were 0.836 and 0.818 , respectively, of a time-dependent receiver operator
\end{abstract}

Correspondence to: Dr Yanxia Zhang, Department of Respiratory, Dongfang Hospital, Beijing University of Chinese Medicine, 6 Fangxingyuan 1st Block, Beijing 100078, P.R. China

E-mail: zhangyx929@126.com

Abbreviations: AUC, area under the curve; CI, confidence interval; FDR, false discovery rate; GO, Gene Ontology; HR, hazard ratio; KEGG, Kyoto Encyclopedia of Genes and Genomes; K-M analysis, Kaplan-Meier analysis; LASSO, least absolute shrinkage and selection operator; lncRNA, long non-coding RNA; LUAD, lung adenocarcinoma; NSCLC, non-small-cell lung cancer; OS, overall survival; ROC, receive operator characteristic; TCGA, The Cancer Genome Atlas.

Key words: long non-coding RNA, lung adenocarcinoma, prognostic signature, risk score, overall survival, biomarkers characteristic curve. Moreover, lncRNAs AC124804.1 and MIR34AHG were identified using K-M survival analysis and the potential function of these two lncRNAs was predicted using Gene Ontology and Kyoto Encyclopedia of Genes and Genomes functional enrichment. The present lncRNA model provides novel insight which may improve prediction of prognosis for patients with LUAD and identify potentially novel biomarkers for the diagnosis.

\section{Introduction}

Lung cancer is the most common type of malignant tumor and was the leading cause of cancer-associated death in the USA in 2019 (1). Lung cancer can be categorized into two main histological subtypes, non-small-cell lung cancer (NSCLC) and small-cell lung cancer, accounting for $\sim 85 \%$ and $\sim 15 \%$ of cases, respectively $(2,3)$. Lung adenocarcinoma (LUAD) is one of the major subtypes of NSCLC with a low 5-year survival rate of $\sim 20 \%$ in the USA $(4,5)$. It is currently understood that two of the driver oncogenes involved in tumorigenesis and progression of LUAD are epidermal growth factor receptor and anaplastic lymphoma kinase (6-8). These biomarkers can be used for diagnosis and targeted in the treatment of LUAD; however, prognosis remains unsatisfactory due to the underlying molecular heterogeneity and diverse etiology of LUAD. Therefore, it is important that novel valuable biomarkers, which have associations with prognosis in LUAD are identified.

Long non-coding RNA (IncRNA) is a type of non-coding RNA which is $>200$ nucleotides in length (9). Previous research has demonstrated that lncRNAs serve diverse functions in cellular processes and are involved in tumorigenesis, progression and metastasis $(10,11)$. However, only a few lncRNAs involved in the development of LUAD have been identified, including histocompatibility leukocyte antigen complex P5, chromatin-associated RNA 10 and metastasis-associated lung adenocarcinoma transcript 1 (12-14). The underlying molecular mechanisms for the functions of numerous lncRNAs remains unclear, to the best of our knowledge, but it is hypothesized that lncRNAs may have valuable clinical applications in the future.

Previous studies have described several novel lncRNAs with potential use as biomarkers differentially expressed in human LUAD tissue $(15,16)$ and further investigation into these lncRNAs may elude their prognostic value. Integrated prognostic analysis of lncRNAs combined with overall outcomes, 
pathological stages and other clinical parameters may improve the prediction of overall survival time of patients with LUAD.

In the present study, the construction method for a previous lncRNA gene signature was amended $(17,18)$, aiming to improve its accuracy. Based on lncRNA expression profiling, a prognostic model was constructed using a comprehensive approach involving the least absolute shrinkage and selection operator (LASSO) algorithm and multivariate Cox regression. Model robustness and accuracy were assessed using area under the calculated curve (AUC) and Kaplan-Meier (K-M) survival analyses in high- and low-risk groups. Finally, potential biomarkers associated with survival status were identified using K-M survival analysis and possible functions of these biomarkers were predicted using enrichment analysis of co-expressed mRNAs.

\section{Materials and methods}

Datasets. RNA sequencing data and corresponding clinical information were obtained from The Cancer Genome Atlas (TCGA) database (cancer.gov/tcga), including data for 535 LUAD tissue samples and 59 control adjacent normal tissues. The LUAD group consisted of 249 males and 286 females with an age range of 33-88 years and a median age of 66 years, whereas the control group consisted of 25 males and 34 females with an age range of 42-86 years and a median age of 66 years. LUAD patients with incomplete survival data were excluded, therefore 500 samples were used for analysis.

Identification of differentially expressed lncRNAs. All analyses were performed using $\mathrm{R}$ software (version 3.5.3; r-project.org/) and $R$ packages by $R$ studio version 1.1.463 $(19,20)$. Raw lncRNA expression profiles were constructed using an expression matrix and normalized using the edgeR package (version 3.22.5) (21) and the lncRNAs differentially expressed between LUAD and control samples were identified. IncRNAs which met the threshold of $\log 2$ [fold change (FC)] $\geq 2$ and false discovery rate $(\mathrm{FDR})<0.05$ were screened out for subsequent analysis.

Definition of the gene-related prognostic model. Univariate Cox, LASSO and multivariate Cox regression analyses were performed to identify the association between survival time and lncRNA expression using the glmnet (version 2.0.18) (22) and survival (version 2.44.1.1) (23) packages. A univariate Cox model was established to assess the correlation between overall survival (OS) and lncRNA expression levels. $\mathrm{P}<0.05$ was considered to indicate a statistically significant difference. lncRNAs meeting this criterion were included in LASSO regression analysis, which was to further select prognostic genes and to avoid the over-fitting of the signature model. After selection using the minimum $\lambda$ value, which represented the optimal number of variables in this model, a further refined group of lncRNAs was included in a multivariate Cox analysis to determine the independent contribution of each IncRNA to prognosis and hazard ratios (HR) and 95\% confidence intervals $(\mathrm{CI})$ were calculated. The Cox regression coefficient $(\beta)$ and expression levels of lncRNAs were used to calculate prognosis-related risk scores according to the formula: $\Sigma$ (explncRNAn x $\beta$ lncRNAn). Samples were further divided into high- and low-risk groups using the median risk score.

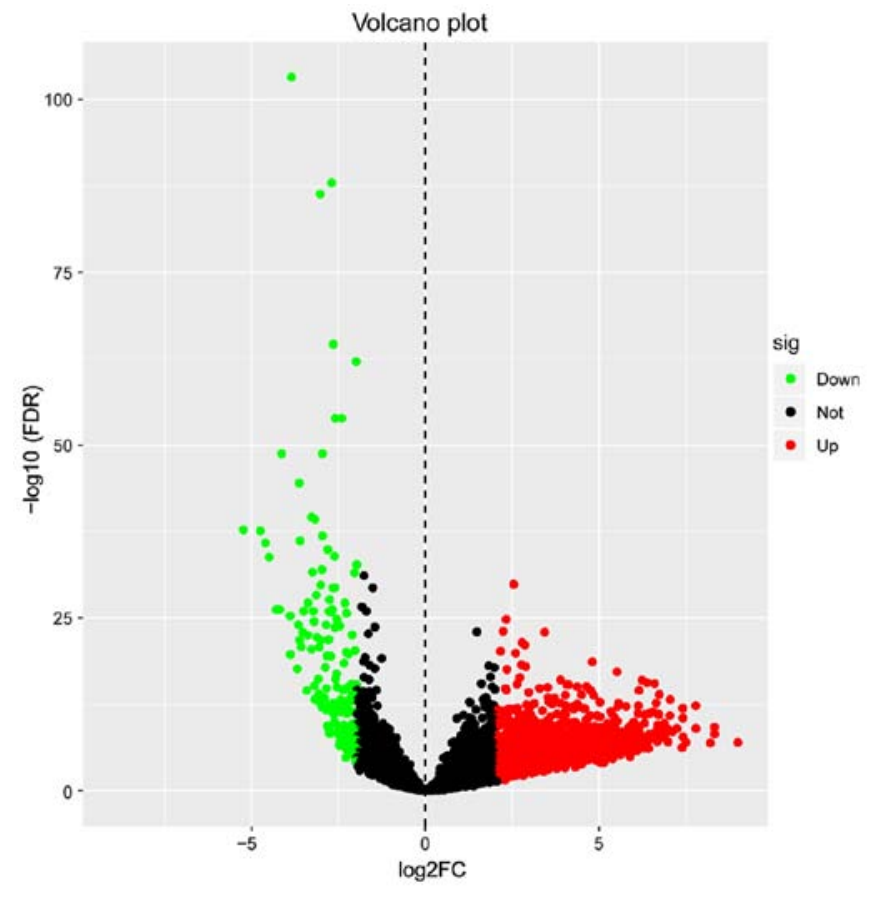

Figure 1. Identification of 1,684 differentially expressed lncRNAs. Red dots represent 1,499 upregulated lncRNAs, green dots represent 185 downregulated lncRNAs and black dots represent 9,736 non-differentially expressed lncRNAs. lncRNA, long non-coding RNA; FC, fold change; sig, significance; down, downregulated; not, not significant; up, upregulated; FDR, false discovery rate.

Evaluation of the risk model and identification of potential biomarkers. Time-dependent receiver operator characteristic (ROC) curves were plotted to evaluate performance of the prognostic model. K-M survival analyses were performed to predict survival times for the high and low risk groups and to calculate AUCs value for the prognostic model. Multivariate Cox analysis results were used to identify potential biomarkers. A threshold of $\mathrm{P}<0.05$ was used to identify eligible lncRNAs for survival and K-M curve analyses and to predict the OS associated with each IncRNA. Subsequently, potential biomarkers were identified if this $\mathrm{P}<0.05$ criterion from the survival curves was met.

Functional enrichment analysis. The biological function of the lncRNAs with significant P-values for OS in the comparison between the high and low risk groups was predicted using their co-expressed protein-coding mRNAs. The mRNAs that correlated with prognostic lncRNAs were identified using Pearson correlation analysis. An absolute correlation coefficient value $>0.4$ was considered significant. The Database for Annotation, Visualization and Integrated Discovery (DAVID) Bioinformatics Tool (version 6.8) $(24,25)$ was used to perform the Gene Ontology (GO) and Kyoto Encyclopedia of Genes and Genomes (KEGG) functional enrichment analysis.

\section{Results}

IncRNAs are differentially expressed between LUAD and control samples. A total of 1,684 differentially expressed lncRNAs (Table SI) were identified, meeting the conditions of $\mid \log 2$ (FC) $\mid \geq 2$ and FDR $<0.05$. Of these, 1,499 lncRNAs were upregulated and 185 lncRNAs were downregulated (Fig. 1). 

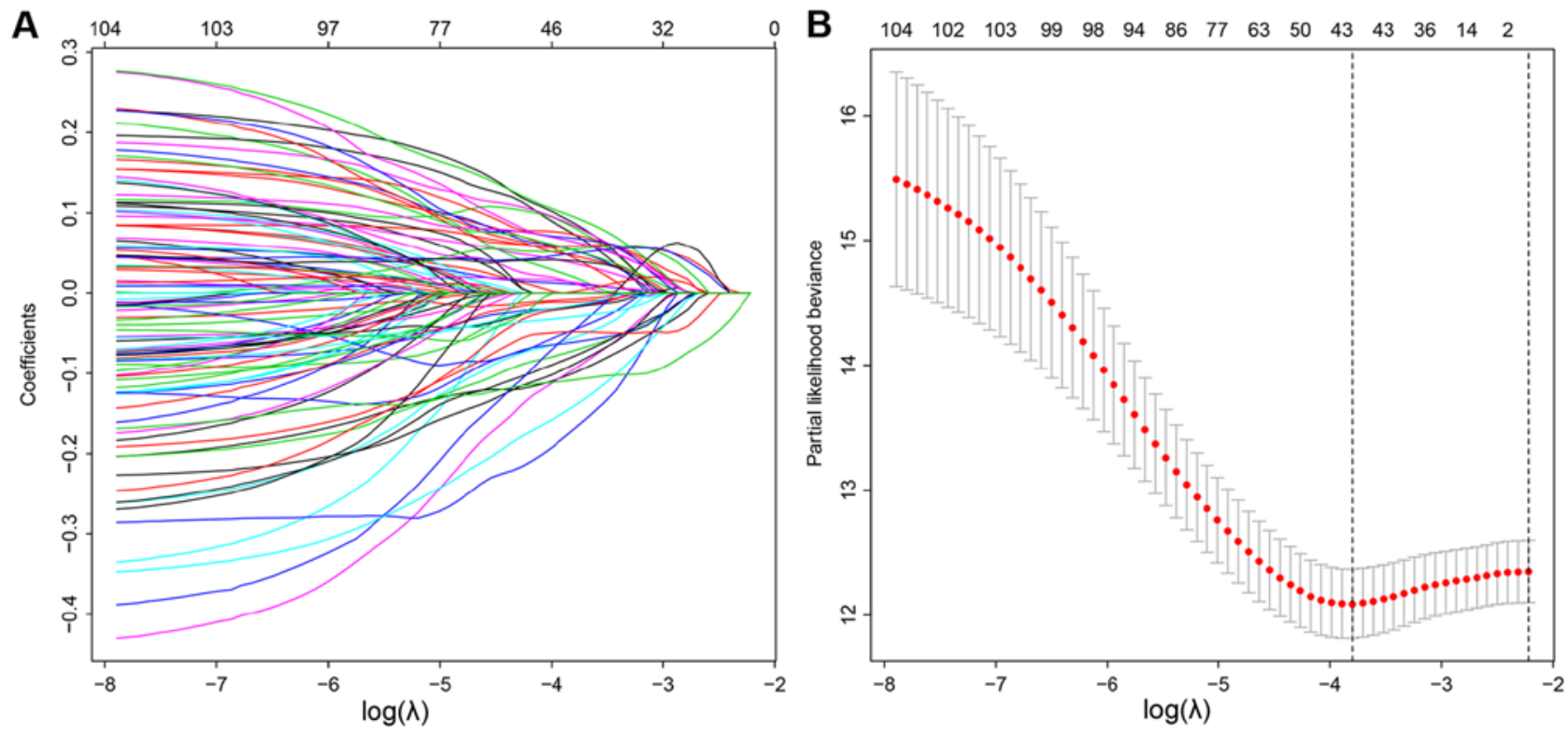

Figure 2. Construction of the prognosis associated molecular signature by LASSO regression. (A) LASSO coefficient profiles of 107 prognostic lncRNAs (B) Tuning parameter selection in the LASSO regression, with vertical lines marking the optimal $\lambda$ value of minimum and 1-SE. LASSO, Least Absolute Shrinkage and Selection Operator; SE, standard error; lncRNA, long non-coding RNA.

The IncRNAs which had significant differential expression levels between the LUAD and control tissue groups were used for subsequent prognostic analysis.

Construction of the prognostic model. Univariate Cox regression was used to analyze the correlations between differentially expressed IncRNA profiles and the OS of patients with LUAD. A total of 107 lncRNAs were significantly correlated with patient OS $(\mathrm{P}<0.05)$ and these IncRNAs were used in a LASSO regression analysis (Table SII). Overall, 44 key lncRNAs were selected when the $\lambda$ value was at a minimum (Fig. $2 \mathrm{~A}$ and $\mathrm{B}$ ). Finally, a multivariate Cox regression analysis was performed to obtain HR values and 95\% CIs for each key lncRNA (Fig. 3). Samples were classified into high- and low-risk groups according to respective median risk scores (Fig. 4A and B).

Evaluation of the prognostic model. K-M survival curves for OS outcomes were performed according to median risk score values, demonstrating that the predicted survival time of the high-risk group was significantly shorter compared with the low risk group $(\mathrm{P}<0.001 ;$ Fig. 5). The AUCs for 3- and 5-year survivals were 0.836 and 0.818 , respectively, of a time-dependent ROC curve demonstrating that the risk score model had stable performance (Fig. 6).

Identification of potential biomarkers. Multivariate Cox regression showed that $\operatorname{lncRNAs}$ with $\mathrm{P}<0.05$ were correlated with survival status (Fig. 3). K-M survival curves enabled screening out of two lncRNAs, AC124804.1 and MIR34AHG, with significantly different predicted survival times between the high- and low-risk score groups (Fig. 7A and B). These two lncRNAs had the greatest correlation with OS time and may have potential as prognostic biomarkers for patients with LUAD.

Functional enrichment. To investigate the underlying molecular mechanisms of the functions of AC124804.1 and
MIR34AHG, the co-expressed mRNAs of these lncRNAs were identified using Pearson's correlation analysis. For MIR34AHG, no mRNAs met the screening condition of a correlation coefficient value of $>0.4$. For AC124804.1, a total of 818 protein coding mRNAs were identified for further functional enrichment. Overall, four GO terms and four KEGG pathways had confirmed association with AC124804.1. The 818 co-expressed mRNAs were primarily clustered in the poly(A) RNA binding and protein binding of molecular functions, and to the mitochondrion and nucleoplasm cellular components $(\mathrm{P}<0.05$; Fig. 8A). KEGG pathway enrichment analysis demonstrated that these mRNAs were primarily associated with Proteasome, Alzheimer's disease, SNARE interactions in vesicular transport and Peroxisome pathways (Fig. 8B).

\section{Discussion}

LUAD is a large global health challenge $(1,26)$ and our knowledge of its underlying mechanisms may be improved by preclinical next-generation sequencing bioinformatics technology $(27,28)$. However, despite advances in the diagnosis and treatment of LUAD, the OS time of patients with LUAD remains poor (29). Recent studies have identified molecular biomarkers throughout the various clinical stages and pathological tissue types $(30,31)$. However, patients with the same clinical and pathological features at diagnosis often have a completely different final prognoses (32). Molecular gene signatures associated with prognosis may aid the identification of novel molecular biomarkers for use in diagnosis and treatment of LUAD in combination with patients' genetic profiles and clinical parameters $(17,18)$. Recent studies pertaining to LUAD have investigated gene signatures based on IncRNA data and the selected lncRNAs may be important risk factors and have potential predictive power for patient survival $(17,18)$. However, due to 


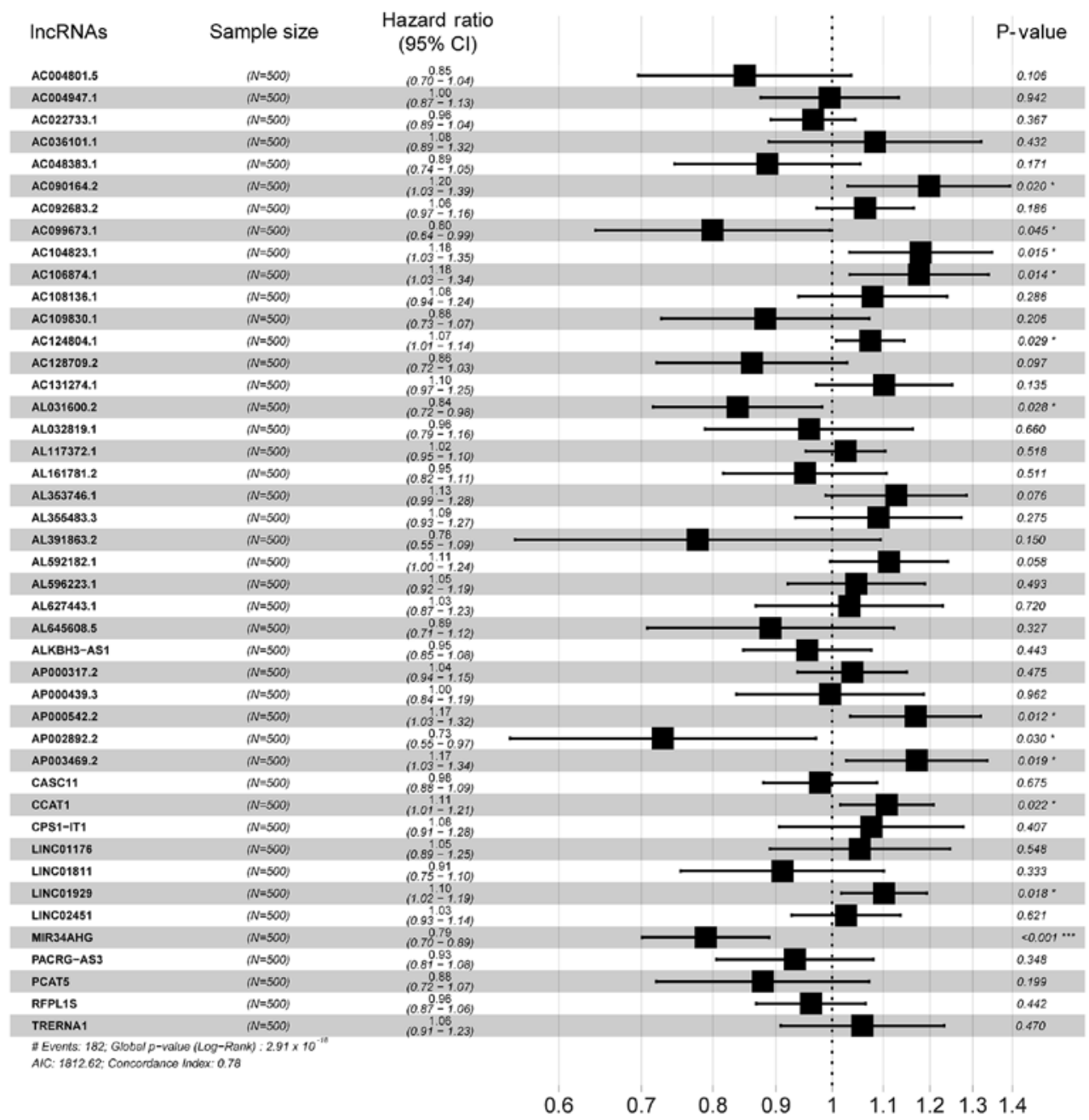

Figure 3. Forest plot demonstrating the HR and $95 \%$ confidence interval for the key lncRNAs. HRs $<1$ represents a negative correlation and HR $>1$ represents a positive correlation. ${ }^{*} \mathrm{P}<0.05 ;{ }^{* *} \mathrm{P}<0.01 ;{ }^{* * *} \mathrm{P}<0.001$. HR, hazard ratio; lncRNA, long non-coding RNA.
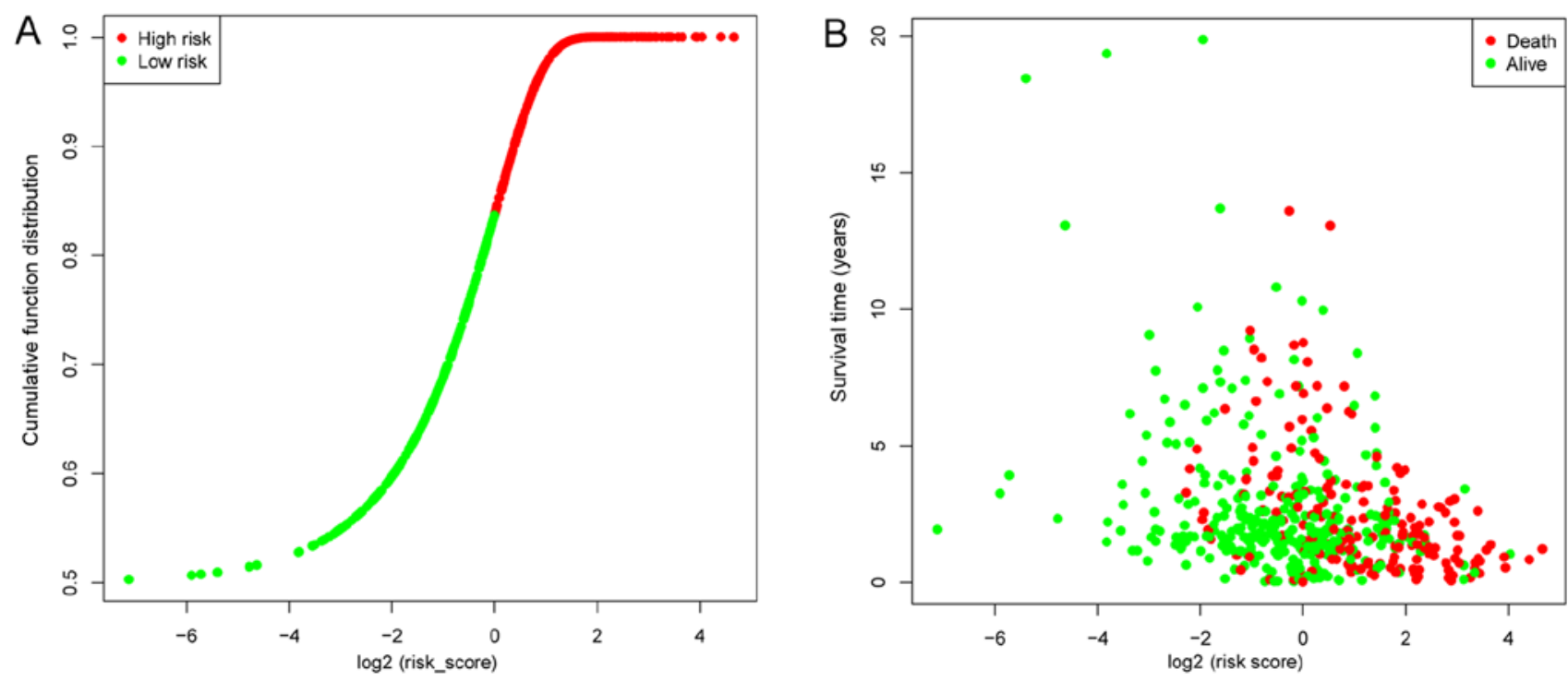

Figure 4. Patient risk scores. (A) Distribution of patient risk scores. Samples were divided into two groups according to the median risk score. (B) Survival status of patients with lung adenocarcinoma. The position of the dots represents the correlation between the survival time and risk scores.

complex underlying mechanisms, the biological functions of the majority of IncRNAs have not been resolved (33).
The prognostic IncRNA signature constructed in the present study, demonstrating correlation with clinical features of 


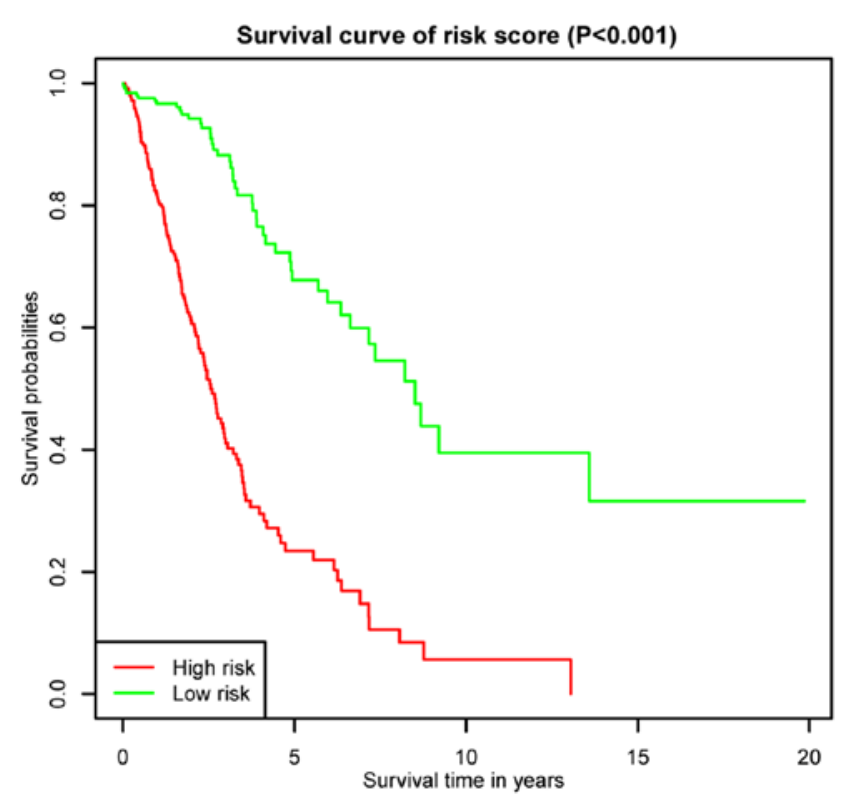

Figure 5. Kaplan-Meier survival curves for overall survival outcomes based on the IncRNA signature. There was a significant difference between the high and low risk groups. IncRNA, long non-coding RNA.

LUAD, may be aid the screening out of potential lncRNAs for further investigation.

To improve the accuracy and robustness of the present model compared with previous studies $(15,17,18)$, a different approach was taken to data preprocessing and construction of the model. First, the present study utilized larger sample sizes compared with previous studies $(15,17,18)$ resulting from differences in inclusion criteria, including a total of 535 LUAD tissues and 59 adjacent normal tissues (controls). Second, the present study used different methods of data preprocessing and normalization. For example, previous studies using some online tools for analysis, whereas the present study performed analysis using edge R package in $\mathrm{R}$ software, which could analyze the latest raw counts of RNA-seq data of TCGA. Third, the present group selected variables using univariate Cox analysis and LASSO regression, which were not employed in previous studies. In addition, the eligible IncRNAs in the present study were confirmed using P-values of multivariate Cox regression and survival analyses.

In the present study, IncRNA profiles were used to construct the prognostic model, using data from tissue samples and clinical information of 500 patients held in the TCGA database. A total of 1,684 differentially expressed lncRNAs were investigated as potential biomarkers using univariate Cox regression to screen out lncRNAs that were significantly associated with the clinical characteristics. The results of univariate Cox regression were further filtered using the LASSO algorithm, which weakens collinearity of the risk model and enables the most influential variables to be selected $(34,35)$. This approach is suitable for dealing with datasets containing a large volume of variables, as occurs in genomics (36). The final $44 \mathrm{lncRNAs}$ identified using LASSO regression were then analyzed using multivariate Cox regression. The correlation of each lncRNA with survival of patients with LUAD was presented as an HR value. Patients were divided into high and low risk groups according to the median risk scores which demonstrated that

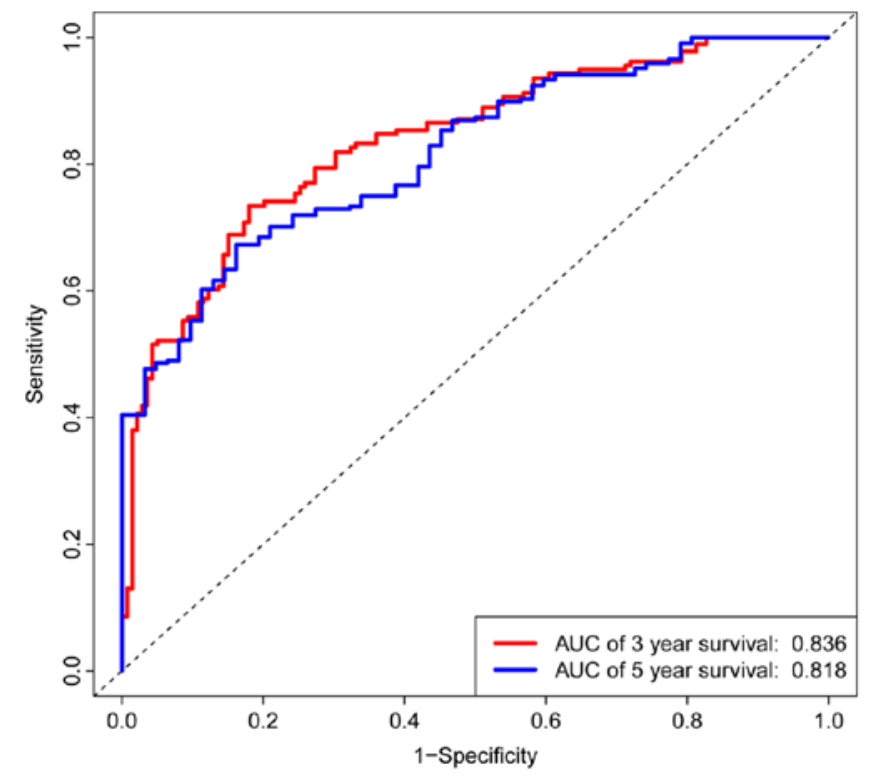

Figure 6. Time-dependent receiver operator characteristic curves for overall survival prediction at 3 - and 5-years. The signature had a moderately stable performance for prognosis prediction. AUC, area under curve.

3 and 5 -year survival times were significantly different between the high and low risk score groups. These data combined with the AUC score results confirmed the accuracy and robustness of this model for predicting prognosis of patient with LUAD.

A multivariate Cox regression forest plot identified lncRNAs with significant P-values as potential prognostic biomarkers for LUAD. The respective survival times correlated with these lncRNAs were calculated prospectively using K-M survival analysis, identifying candidate prognostic factors. According to the prognostic signature constructed in the present study, the expression levels of AC124804.1 and MIR34AHG were significantly correlated with differential clinical outcomes and predicted OS times of the two risk groups. These two lncRNAs may have value as novel biomarkers for estimating prognosis of patients with LUAD. To the best of our knowledge, there is no published work describing the relationships between these IncRNAs and LUAD to date. To explore the potential functions of AC124804.1 and MIR34AHG, the co-expressed protein-coding mRNAs of these lncRNAs were investigated using a Pearson correlation coefficient test followed by GO and KEGG functional enrichment analyses. These analyses demonstrated that the potential underlying molecular mechanisms of AC124804.1 function were pathways involving proteasome, peroxisome and SNARE interactions in vesicular transport, which have confirmed roles in tumorigenesis and progression in previous studies (37-39). MIR34AHG is the host gene of miR-34a and consequently may have biological functions associated with the miR-34 family, which may have suppressive functions in LUAD as previously demonstrated $(40,41)$. The relationship between these two lncRNAs and LUAD should be studied further.

There are several limitations to the present study. First, the data for the lncRNA signatures were from the TCGA database and these samples primarily originated from Caucasians and African Americans, limiting extrapolation of the results of the present study to other ethnic groups. Second, the present study used a database mining design without validation in 

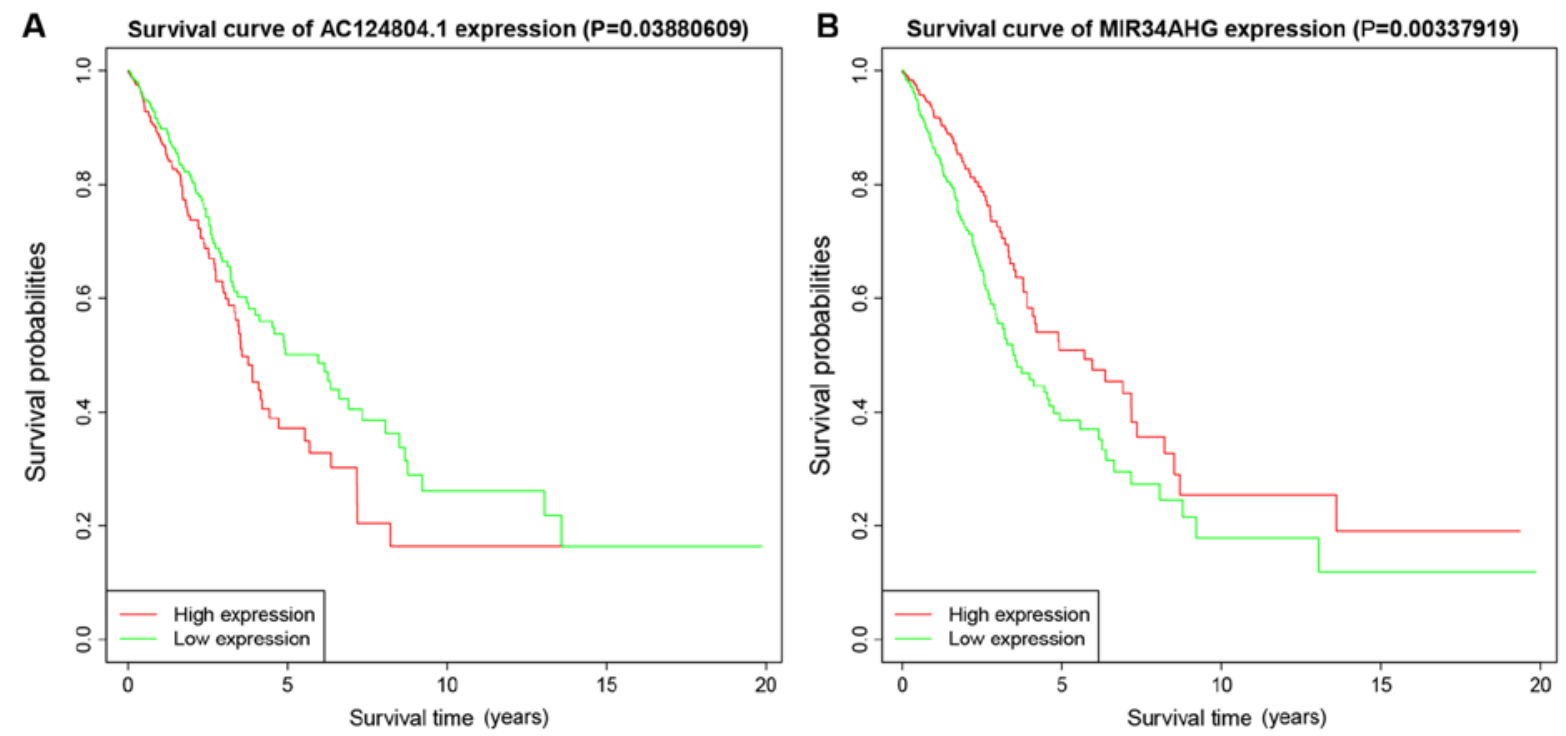

Figure 7. Kaplan-Meier survival curves for overall survival outcomes. Predicted survival time of: (A) AC124804.1 and (B) MIR34AHG long non-coding RNAs.
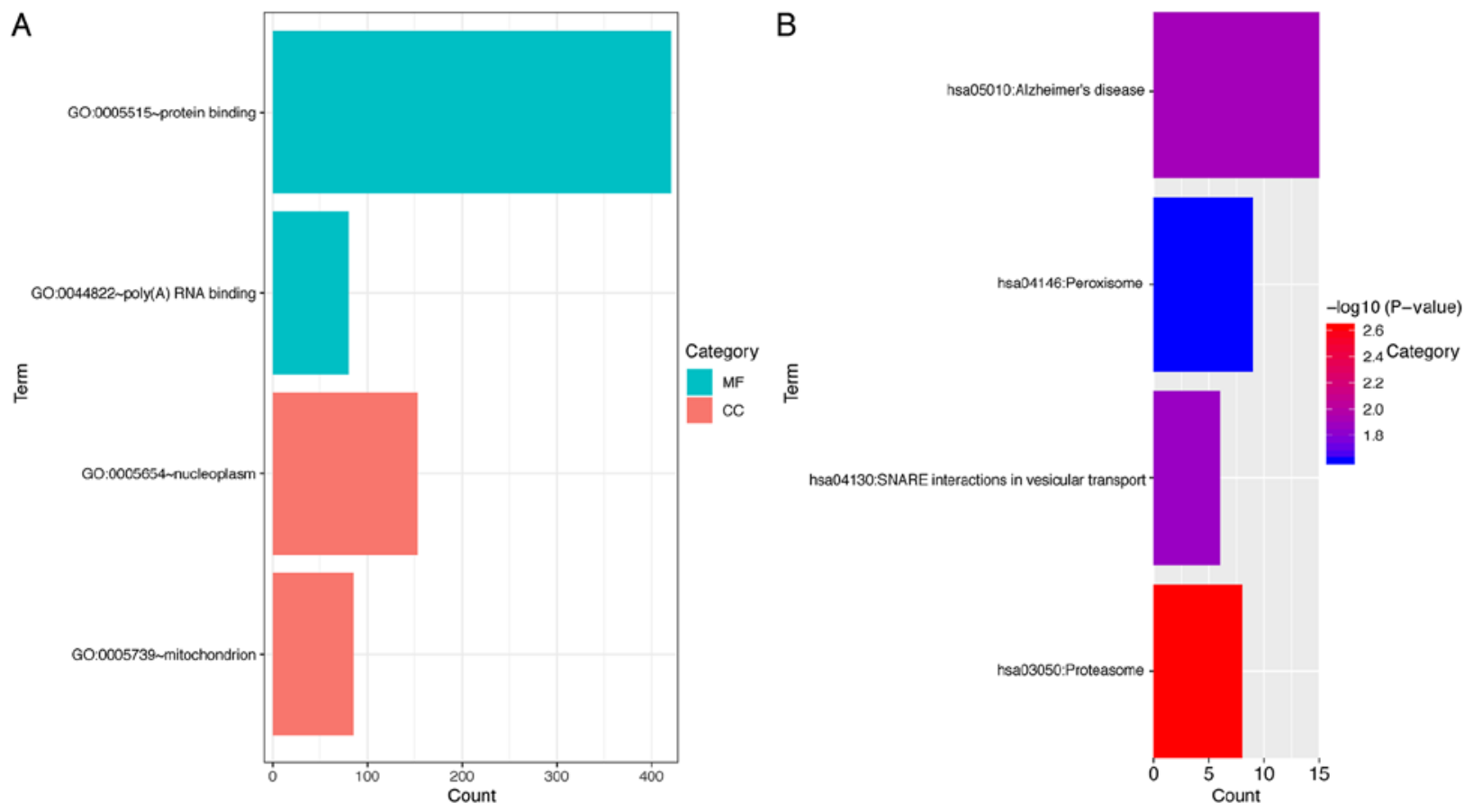

Figure 8. Functional enrichment analysis for the co-expressed protein-coding mRNAs of AC124804.1. (A) Significantly enriched GO terms. The bar lengths indicate the counts enriched in this category. (B) Significantly enriched Kyoto Encyclopedia of Genes and Genomes pathways. The bar lengths indicate the counts enriched in these pathways. GO, Gene Ontology; MF, molecular function; CC, cellular component.

fresh samples and prospective experimental studies. Third, the clinical parameters included only OS time, with histological subtypes and other risk factors for LUAD were excluded from the analysis. The primary purpose of the present study was to identify novel biomarkers for the diagnosis and treatment of LUAD. The lncRNA prognosis signature should be further investigated incorporating more specific clinical characteristics to fully understand the associations involved.

In conclusion, a robust lncRNA signature that could stratify and predict survival time of patients with LUAD was constructed. Furthermore, Cox regression was used to identify two novel survival associated IncRNA biomarkers and provided potential targets for diagnostic and therapeutic application. The biological functions of the two lncRNAs identified in the present study need confirmation in further experimental studies.

\section{Acknowledgements}

Not applicable.

\section{Funding}

The present study was funded by The National Natural Science Foundation of China (grant no. 81603577). 


\section{Availability of data and materials}

The datasets used and/or analyzed during the present study available from the corresponding author on reasonable request.

\section{Authors' contributions}

YZ conceived and designed the study. XY conducted the analysis and wrote the manuscript. All authors read and approved the final manuscript.

\section{Ethics approval and consent to participate}

Not applicable.

\section{Patient consent for publication}

Not applicable.

\section{Competing interests}

The authors declare that they have no competing interests.

\section{References}

1. Siegel RL, Miller KD and Jemal A: Cancer statistics, 2019. CA Cancer J Clin 69: 7-34, 2019.

2. Simó M, Vaquero L, Ripollés P, Gurtubay-Antolin A, Jové J, Navarro A, Cardenal F, Bruna J and Rodríguez-Fornells A: Longitudinal brain changes associated with prophylactic cranial irradiation in lung cancer. J Thorac Oncol 11: 475-486, 2016.

3. Shahabi S, Kumaran V, Castillo J, Cong Z, Nandagopal G, Mullen DJ, Alvarado A, Correa MR, Saizan A, Goel R, et al LINC00261 is an epigenetically regulated tumor suppressor essential for activation of the DNA damage response. Cancer Res 79: 3050-3062, 2019.

4. Lu GS, Li M, Xu CX and Wang D: APE1 stimulates EGFR-TKI resistance by activating Akt signaling through a redox-dependent mechanism in lung adenocarcinoma. Cell Death Dis 9: 1111, 2018

5. Macheleidt IF, Dalvi PS, Lim SY, Meemboor S, Meder L, Käsgen O, Müller M, Kleemann K, Wang L, Nürnberg P, et al: Preclinical studies reveal that LSD1 inhibition results in tumor growth arrest in lung adenocarcinoma independently of driver mutations. Mol Oncol 12: 1965-1979, 2018.

6. Hung MS, Lung JH, Lin YC, Fang YH, Huang SY, Jiang YY, Hsieh MJ and Tsai YH: Comparative analysis of two methods for the detection of EGFR mutations in plasma circulating tumor DNA from lung adenocarcinoma patients. Cancers (Basel) 11: 803, 2019.

7. Li S, Choi YL, Gong Z, Liu X, Lira M, Kan Z, Oh E, Wang J, Ting JC, Ye X, et al: Comprehensive characterization of oncogenic drivers in Asian lung adenocarcinoma. J Thorac Oncol 11: 2129-2140, 2016.

8. Wang X, Min S, Liu H, Wu N, Liu X, Wang T, Li W, Shen Y, Wang H, Qian Z, et al: Nf1 loss promotes Kras-driven lung adenocarcinoma and results in Psat1-mediated glutamate dependence. EMBO Mol Med 11: e9856, 2019.

9. Zhang L, Meng X, Zhu XW, Yang DC, Chen R, Jiang Y and Xu T: Long non-coding RNAs in Oral squamous cell carcinoma: Biologic function, mechanisms and clinical implications. Mol Cancer 18: 102, 2019.

10. Yan Y, Xu Z, Li Z, Sun L and Gong Z: An insight into the increasing role of lncRNAs in the pathogenesis of gliomas. Front Mol Neurosci 10: 53, 2017.

11. Xu Z, Yan Y, Qian L and Gong Z: Long non-coding RNAs act as regulators of cell autophagy in diseases (Review). Oncol Rep 37: 1359-1366, 2017.

12. Jiang L, Wang R, Fang L, Ge X, Chen L, Zhou M, Zhou Y, Xiong W, Hu Y, Tang X, et al: HCP5 is a SMAD3-responsive long non-coding RNA that promotes lung adenocarcinoma metastasis via miR-203/SNAI axis. Theranostics 9: 2460-2474, 2019.
13. Ge X, Li GY, Jiang L, Jia L, Zhang Z, Li X, Wang R, Zhou M, Zhou Y, Zeng Z, et al: Long noncoding RNA CAR10 promotes lung adenocarcinoma metastasis via miR-203/30/SNAI axis. Oncogene 38: 3061-3076, 2019.

14. Chen J, Liu X, Xu Y, Zhang K, Huang J, Pan B, Chen D, Cui S, Song H, Wang R, et al: TFAP2C-activated MALAT1 modulates the chemoresistance of docetaxel-resistant lung adenocarcinoma cells. Mol Ther Nucleic Acids 14: 567-582, 2019.

15. Salavaty A, Rezvani Z and Najafi A: Survival analysis and functional annotation of long non-coding RNAs in lung adenocarcinoma. J Cell Mol Med 23: 5600-5617, 2019.

16. Serghiou S, Kyriakopoulou A and Ioannidis JP: Long noncoding RNAs as novel predictors of survival in human cancer: A systematic review and meta-analysis. Mol Cancer 15: 50, 2016.

17. Shi X, Tan H, Le X, Xian H, Li X, Huang K, Luo VY, Liu Y, $\mathrm{Wu} \mathrm{Z,} \mathrm{Mo} \mathrm{H,} \mathrm{et} \mathrm{al:} \mathrm{An} \mathrm{expression} \mathrm{signature} \mathrm{model} \mathrm{to} \mathrm{predict}$ lung adenocarcinoma-specific survival. Cancer Manag Res 10: 3717-3732, 2018

18. Sui J, Yang S, Liu T, Wu W, Xu S, Yin L, Pu Y, Zhang X, Zhang Y, Shen B, et al: Molecular characterization of lung adenocarcinoma: A potential four-long noncoding RNA prognostic signature. J Cell Biochem 120: 705-714, 2019.

19. R Core Team (2012). R: A language and environment for statistical computing. R Foundation for Statistical Computing, Vienna, URL. http://www.R-project.org/.

20. RStudio Team (2015). RStudio: Integrated Development for R. RStudio, Inc., Boston, MA, URL. http://www.rstudio.com/.

21. Robinson MD, McCarthy DJ and Smyth GK: edgeR: A Bioconductor package for differential expression analysis of digital gene expression data. Bioinformatics 26: 139-140, 2010.

22. Friedman J, Hastie T and Tibshirani R: Regularization paths for generalized linear models via coordinate descent. J Stat Softw 33: 1-22, 2010.

23. Therneau TM and Grambsch PM: Modeling Survival Data: Extending the Cox Model. In: Statistics for Biology and Health Springer Science and Business Media, New York, NY, pp39-76, 2000.

24. Dennis G Jr, Sherman BT, Hosack DA, Yang J, Gao W, Lane HC and Lempicki RA: DAVID: Database for annotation, visualization, and integrated discovery. Genome Biol 4: 3, 2003.

25. Yan Y, Xu Z, Qian L, Zeng S, Zhou Y, Chen X, Wei J and Gong Z: Identification of CAV1 and DCN as potential predictive biomarkers for lung adenocarcinoma. Am J Physiol Lung Cell Mol Physiol 316: L630-L643, 2019.

26. Sivakumar S, San Lucas FA, Jakubek YA, McDowell TL, Lang W, Kallsen N, Peyton S, Davies GE, Fukuoka J, Yatabe Y, et al: Genomic landscape of allelic imbalance in premalignant atypical adenomatous hyperplasias of the lung. EBioMedicine 42: 296-303, 2019

27. Yu C, Tian F, Liu J, Su M, Wu M, Zhu X and Qian W: Circular RNA cMras inhibits lung adenocarcinoma progression via modulating miR-567/PTPRG regulatory pathway. Cell Prolif 52: e12610, 2019.

28. Kerdidani D, Chouvardas P, Arjo AR, Giopanou I, Ntaliarda G, Guo YA, Tsikitis M, Kazamias G, Potaris K, Stathopoulos GT, et al: Wnt 1 silences chemokine genes in dendritic cells and induces adaptive immune resistance in lung adenocarcinoma. Nat Commun 10: 1405, 2019.

29. Misono S, Seki N, Mizuno K, Yamada Y, Uchida A, Sanada H, Moriya S, Kikkawa N, Kumamoto T, Suetsugu T, et al: Molecular pathogenesis of gene regulation by the miR-150 duplex: miR-150-3p regulates TNS4 in lung adenocarcinoma. Cancers (Basel) 11: 601, 2019.

30. Abdel-Aziz A, Ahmed RA and Ibrahiem AT: Expression of pRb, Ki67 and HER 2/neu in gastric carcinomas: Relation to different histopathological grades and stages. Ann Diagn Pathol 30: 1-7, 2017.

31. Ren Y, Zhao S, Jiang D, Feng X, Zhang Y, Wei Z, Wang Z, Zhang W, Zhou QF, Li Y, et al: Proteomic biomarkers for lung cancer progression. Biomarkers Med 12: 205-215, 2018.

32. Wang P, Jin M, Sun CH, Yang L, Li YS, Wang X, Sun YN, Tian LL and Liu M: A three-lncRNA expression signature predicts survival in head and neck squamous cell carcinoma (HNSCC). Biosci Rep 38: BSR20181528, 2018.

33. Peng W, He D, Shan B, Wang J, Shi W, Zhao W, Peng Z, Luo Q, Duan M, Li B, et al: LINC81507 act as a competing endogenous RNA of miR-199b-5p to facilitate NSCLC proliferation and metastasis via regulating the CAV1/STAT3 pathway. Cell Death Dis 10: 533, 2019. 
34. Machado AD, Gómez LM, Marchioni DM, Dos Anjos FS, Molina MD, Lotufo PA, Benseñor IJ and Titan SM: Association between dietary intake and coronary artery calcification in non-dialysis chronic kidney disease: The PROGREDIR Study. Nutrients 10: 372, 2018.

35. Kwon Y, Lee T, Lang A and Burnette D: Assessment on latitudinal tree species richness using environmental factors in the southeastern United States. PeerJ 7: e6781, 2019.

36. Waldmann P, Ferenčaković M, Mészáros G, Khayatzadeh N, Curik I and Sölkner J: AUTALASSO: An automatic adaptive LASSO for genome-wide prediction. BMC Bioinformatics 20: $167,2019$.

37. Follo C, Vidoni C, Morani F, Ferraresi A, Seca C and Isidoro C: Amino acid response by Halofuginone in cancer cells triggers autophagy through proteasome degradation of mTOR. Cell Commun Signal 17: 39, 2019.

38. Kaur S, Nag A, Gangenahalli G and Sharma K: Peroxisome proliferator activated receptor gamma sensitizes non-small cell lung carcinoma to gamma irradiation induced apoptosis. Front Genet 10: 554, 2019.
39. Wang YS, Tzeng HT, Tsai CH, Cheng HC, Lai WW, Liu HS and Wang YC: VAMP8, a vesicle-SNARE required for RAB37-mediated exocytosis, possesses a tumor metastasis suppressor function. Cancer Lett 437: 79-88, 2018.

40. Yun MR, Lim SM, Kim SK, Choi HM, Pyo KH, Kim SK, Lee JM Lee YW, Choi JW, Kim HR, et al: Enhancer remodeling and MicroRNA alterations are associated with acquired resistance to ALK inhibitors. Cancer Res 78: 3350-3362, 2018.

41. Zhao K, Cheng J, Chen B, Liu Q, Xu D and Zhang Y: Circulating microRNA-34 family low expression correlates with poor prognosis in patients with non-small cell lung cancer. J Thorac Dis 9: 3735-3746, 2017.

This work is licensed under a Creative Commons Attribution-NonCommercial-NoDerivatives 4.0 International (CC BY-NC-ND 4.0) License. 